\title{
Disclosures of illness by doctors to their patients: A qualitative study of doctors with HIV and other serious disorders
}

\author{
Robert Klitzman $^{\text {a,* }}$, Jonathan Weiss ${ }^{\mathrm{b}, 1}$ \\ ${ }^{a}$ HIV Center for Clinical and Behavioral Studies, New York State Psychiatric Institute and Columbia University, \\ 1051 Riverside Drive, Unit 15, New York, NY 10032, United States \\ ${ }^{\mathrm{b}}$ University of Illinois College of Medicine, Urbana, IL, United States
}

Received 10 December 2005; received in revised form 1 March 2006; accepted 3 March 2006

\begin{abstract}
Objective: To examine issues concerning doctor's disclosures of their illness to their patients.

Methods: We interviewed 50 health care providers who had serious illnesses concerning their experiences with disclosures of their illness to patients.

Results: With regard to their diagnoses, these doctors struggled with whether, when, how and what to tell patients. These issues were prominent, and had broader implications for doctor-patient communication and interactions among doctors with HIV, but arose among doctors with other diagnoses as well. Particularly with HIV, questions emerged concerning whether to: tell patients without being asked, respond only if asked, tell the truth, lie or misrepresent the information. Patients appeared to face dilemmas of whether to ask about a doctor's diagnosis, and whether they had a right to know. Some patients hesitated to ask or felt ambivalent about knowing, as the illness could threaten the doctor-patient relationship. At times, patients learned of a doctor's illness only after the latter had died. Disclosures could strengthen or skew the doctor-patient relationship. We present a model and framework - concerning the complexities of these communications - that can be useful in exploring other key aspects of doctor-patient interactions.

Conclusion: These data raise larger questions of what information patients should be told about physicians. Medical education needs to address these issues better.

Practice implications: Physicians should realize that patients may be anxious about these concerns, and may view the pros and cons of physicians' disclosures of illness differently than do these physicians themselves.
\end{abstract}

(C) 2006 Elsevier Ireland Ltd. All rights reserved.

Keywords: Doctor-patient communication; Doctor-patient relationships; Disclosure; Trust; Decision-making; Qualitative research

\section{Introduction}

Physicians who become ill confront difficult questions of whether, when, and what to disclose concerning their illness to their patients. These decisions may potentially affect doctor-patient relationships and communication, and patient satisfaction and trust. Hence treatment access, utilization, adherence, and coping can be influenced as well

\footnotetext{
* Corresponding author. Tel.: +1 212543 3710; fax: +1 2125436003 .

E-mail addresses: rlk2@columbia.edu (R. Klitzman),weiss1@uiuc.edu (J. Weiss)

${ }^{1}$ Tel.: +1 2173337978 .
}

[1-3]. Previous work has suggested that among patients with HIV, those who are also health care workers may differ from others in coping and disclosure [4,5].

Doctors have a right to privacy concerning their personal health information. Yet patients have a right to be given information that is necessary to provide informed consent (e.g., concerning treatment risks and benefits). Thus, if a physician's illness can potentially harm a patient significantly, these principles can conflict. For HIV, e.g., some have argued that a physician's disclosure of his or her illness may be relevant in exposure-prone procedures [6]. But what about in other situations? Do on-going continuity of care and trust ever warrant disclosure of a doctor's illness? Do these 
decisions affect doctor-patient relationships, and if so, how? How are these issues viewed and approached in clinical situations?

Distinct roles of doctor and patient have been described [7], but it is not clear what happens when these blur-that is, when doctors themselves become patients. An individual with a stigma seeks to "pass" as "untainted" [8]. But how do physicians do so in the context of doctor-patient relationships, given the distinct roles of healer and patient?

These questions concerning illness disclosure have not been systematically examined. Doctors who become patients have been discussed, essentially anecdotallyusually individual doctors publishing accounts of experiences with particular disorders [9-15]. These reports have generally focused on other areas such as increased awareness of the authoritarianism of other physicians $[16,17]$. Disclosures by patients to providers and others have also been examined [5]. Doctors' disclosure of personal information has been probed, focusing on casual remarks regarding their own attitudes and feelings toward treatment (e.g., "I wish I could sleep standing") [18]. Following such disclosures, patient satisfaction varied with whether the discloser was an internist or a surgeon [19]. Physician disclosure of health behaviors (e.g., diet and exercise) can also motivate patients concerning these behaviors [20]. But prior studies did not explore physicians' disclosures of their own serious or potentially fatal illnesses, or explicit medical problems-more sensitive areas that may threaten the stability of the doctor-patient relationships, and thus be approached very differently.

Psychotherapists' disclosures to patients of personal characteristics (e.g., religion and marital status) have been discussed as well, and remain controversial. A psychotherapist's pregnancy, e.g., can evoke a wide range of psychological responses [21]. But disclosure of serious illness has not been examined in these contexts $[22,23]$. Moreover, psychotherapy differs in fundamental ways from encounters between non-psychiatrist physicians and patients concerning medical information. In the former, doctorpatient relationships, transference and countertransference can be critical factors in treatment. Impaired physicians (e.g., due to substance abuse) have received attention, too, as such impairments may potentially be important in patients receiving optimal quality of care [24].

In psychiatry, attention has been given to sexual boundary violations, but less to boundaries concerning self-disclosure. Some argue that a slippery slope exists of disclosures of doctors' personal problems, potentially leading to sexual involvement, and that "self-disclosure is itself a boundary problem because it is a misuse of the patient to satisfy one's own needs for comfort or sympathy" [25]. Others point out a lack of data as to such dangers of self-disclosure, and the importance of motivations and contexts, since some therapist self-disclosure may be appropriate [26]. Hence, egregious versus benevolent therapeutic disclosures need to be differentiated.
Systematic research is thus needed to explore physicians' disclosures of illness to patients and key questions of how these issues are viewed and approached in the complex dynamics of doctor-patient relationships.

\section{Methods}

Pilot interviews were first conducted about issues concerning physicians who become patients. These interviews led to the development and refinement of an instrument. The full study focused in the initial stage on HIV-infected doctors, and was then expanded to include physicians with other diagnoses, too. Subjects were recruited for the full study through emailed announcements (e.g., stating, "Are you or do you know a physician with a serious illness?"), websites, word of mouth, and ads in newsletters. The PI was then contacted by 48 doctors, 1 dentist, and 1 medical student who had become patients due to serious illnesses (referred to below as "doctors"). Two in-depth, semi-structured interviews of $2 \mathrm{~h}$ were held with each subject concerning experiences before and after diagnosis. Serious illness was self-defined, and then confirmed by the PI. Of these participants, 27 were HIV positive, and 23 had other medical problems (e.g., cancer, heart disease, and hepatitis). Ages ranged from 25 to 87, all were Caucasian, except for 1 Latino doctor, 40 were men, and 10 were women. They were interviewed in several cities in participants' homes or offices, or the PI's office-whatever was more convenient for them. Participants were asked about experiences as patients and as providers, and about other aspects of their lives.

Interviews were audiotaped, transcribed, and contentanalyzed, informed by grounded theory [27]. Initial analyses were conducted during the period in which the interviews were being held. A research team, composed of the PI and a research assistant, examined a subset of interviews to assess factors that shaped subjects' experiences, identifying categories of recurrent themes and issues that were subsequently given codes. A senior consultant with expertise in qualitative research provided input at several stages of this coding process. The team assessed similarities and differences between participants, examining categories that emerged, ranges of variation within categories, and variables that may be involved. A coding manual was developed, and areas of disagreement were examined until consensus was reached. New themes that did not fit into this original coding framework were discussed, and modifications were made in the manual when deemed appropriate. In phase two of the analysis, the research team refined, merged, or subdivided thematic categories into secondary or sub-codes, when suggested by associations or overlap in the data. These codes and sub-codes were then used in analysis of all of the interviews. To ensure coding reliability, all interviews were analyzed by two coders.

The study was approved by the Institutional Review Board at Columbia University and the New York State Psychiatric Institute. 


\section{Results}

Several patterns of themes and issues emerged, as depicted in Fig. 1. As described below, subjects struggled with whether and what to disclose concerning their own health status and personal life to patients, and these subjects observed that patients approached the possibility of a doctor being ill in various ways. These issues arose prominently among doctors with HIV, but also appeared among doctors with other disorders. In the case of HIV - given that it is infectious, carries particular stigma, and constitutes an epidemic, affecting many others - these issues can have broad implications for doctor-patient communication and relationships. Doctor-patients, particularly those with any potentially life-threatening disorder (e.g., cancer), confronted similar questions, though at times to lesser degrees. Still, we have decided to present the experiences of doctors with both sets of disorders, in order to provide as full a sense as possible of similarities and differences that arose concerning these issues.

\subsection{Patients' decisions about asking}

Patients may observe evidence of disease in a doctor, and then have to decide whether to ask about his or her health. This issue arose when symptoms of the illness were visible and appeared to be serious - for both HIV and other conditions. A pediatrician with cancer, e.g., became bald, leading to patients' inquiries about his health, and more of a human-tohuman, as opposed to doctor-to-patient, relationship:

Since my hair was gone, patients were aware of it. I couldn't hide it. They'd ask how I was, and it deepened the relationship. I didn't tell every patient. I didn't want them to worry. But if they seemed to notice, I would tell them: "I'm on chemo, and I'm doing well". The message was: "It's not a big problem".

He was not ethically obligated to disclose, but did so within the complex interpersonal dynamics of these relationships, responding to questions that surfaced.

With HIV, given the stigma involved, these concerns had broader implications; patients might sense that discussion was off-limits, and hence reveal concerns about a doctor's health only afterwards or indirectly. A radiologist with HIV said, "People have said things without asking ... 'Doctor, I'm so glad you look so much better. We were really worried that something really bad was going on!"'

Patients, especially if new, often were seen as seeking long-term relationships with providers, and wanting to know

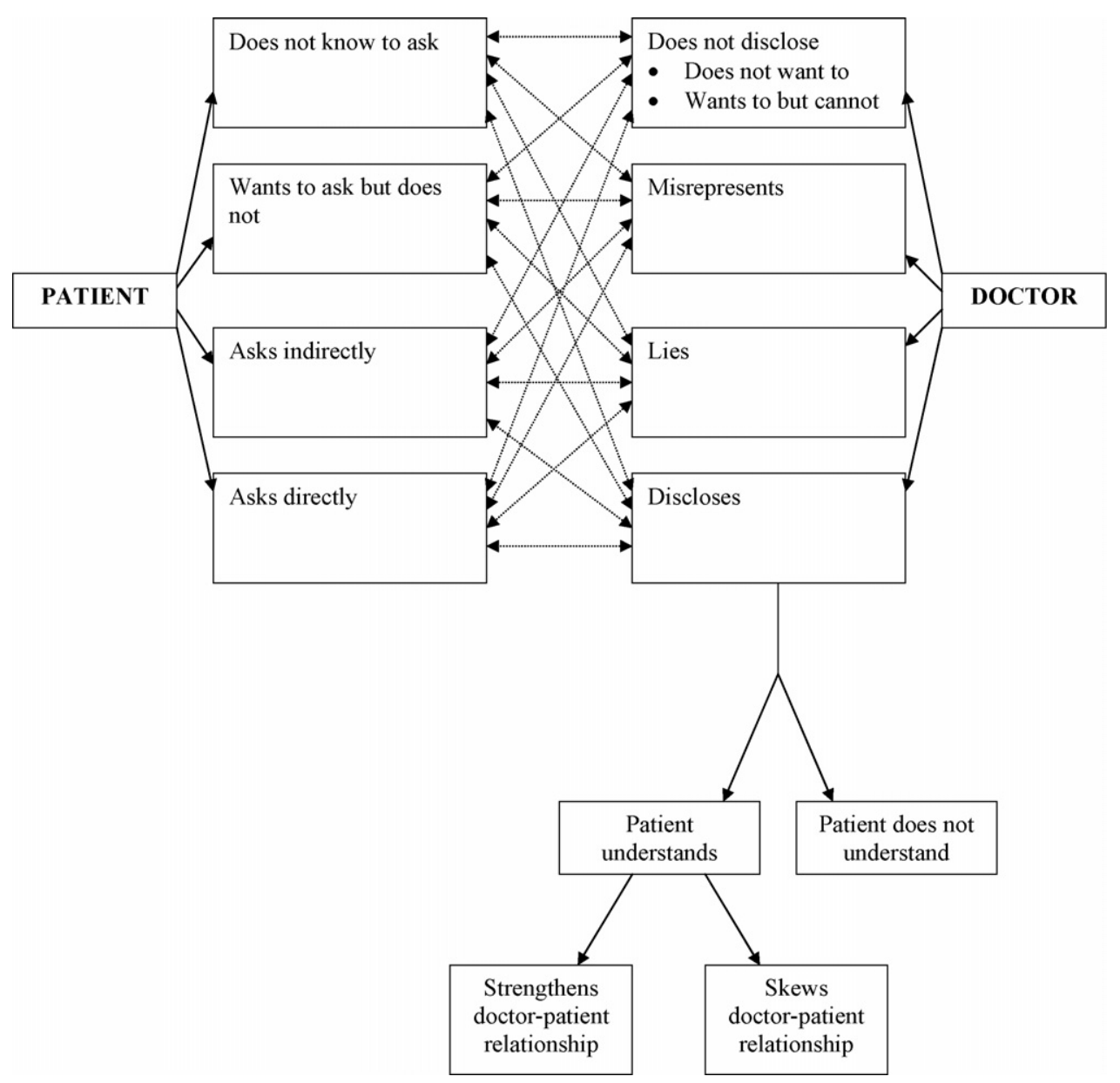

Fig. 1. Summary of patterns of communication between doctors and patients concerning doctor's illness. 
that their doctor would be available over time. A psychiatrist with HIV felt confidentiality could be a problem because of patients wanting such continuity.

One woman's son had died of AIDS ... she said, "I'm so glad to meet you and see how young you are because I never want to change psychiatrists again!"

Still, patients may wonder about a physician's health status, but not raise the topic. An HIV-infected doctor in a medium-sized city said patients questioned him about other doctors. "It comes up with all the doctors in town: people questioning whether they're positive or not. The only time I would address it would be if rumors were false or obstructing patients' interactions with me".

Patients may wonder whether a physician is ill, but feel too awkward or uncomfortable to ask. Such personal inquiry could feel "taboo". Thus, patients may not dare to ask, but want to be told. The patients of one doctor with HIV would not ask about this doctor's health, but waited to be informed about it. Such reticence may have resulted from patients' own fear and denial of a physician being ill or dying.

This doctor had K.S. on his face, and patients wouldn't ask. They were just waiting for him to tell them. After he was hospitalized, people started to come forward and ask. But the staff wouldn't answer. It might have been their denial, too, even though the guy had lost 30 pounds and looked like hell.

Yet, given the stigma of HIV, patients may inquire in ways, or at times, that a doctor deems inappropriate. A gay internist with HIV, e.g., was once asked rudely about his health:

A straight woman in her late $30 \mathrm{~s}$ with the flu walked in off the street with her boyfriend-white-trash type. But regardless, I wasn't just going to give her antibiotics and send her out, that's not the way I practice medicine ... So I asked her if she had ever been tested for HIV. She got indignant and said, "Yeah, about 3 years ago". Her boyfriend was there and maybe she was bothered about discussing it. I said, "And it was negative?" She said, "Yeah", and...she turned around and said, "And you? ... Have you been tested?" I said, "Yeah", and she said, "And?" I said, "And I'm positive". Her mouth dropped... I thought: this could come back to haunt me ... But I'd already decided I was not going to live like that ... I'm sure she'll never come back, but that's fine.

Though he disclosed, he feared that to do so was not entirely appropriate or ethical, and that he could face repercussions.

\subsection{Doctors' decisions about disclosure}

Due to a sense of professional boundaries and privacy, many doctors thus struggled to balance the pros and cons of disclosing their illness to patients. A psychiatrist with metastatic breast cancer returned to work after hospitalization, yet questions lingered of what to tell inquiring patients. "Patients were asking, 'What happened? Where is she?' Nobody would say anything. That was a dilemma: what to tell the patients".

She and some other physicians wanted to disclose to patients, but did not have opportunities to do so. After a hospitalization, she had her voicemail and patients taken away, but she still wanted to treat a particular patient. "I still try to see my first long-term patient who was taken away from me, and who I had a very good relationship with. I really wanted to tell her what happened. I never had the chance. I've been postponed and postponed. People don't want to deal with a sick colleague".

Yet at times, doctors disclosed their disorders - for several different reasons. Most importantly, at times, doctors believed that disclosure about their own experiences, particularly about difficult aspects of treatment, could benefit patients, reducing obstacles to adherence (e.g., resistance to the arduousness of taking pills), or anxieties (e.g., about procedures, general anesthesia and surgery). Several doctors hence volunteered personal health information to patients, to express empathy or encouragement about these aspects of treatment.

Doctors thus made judgments about whether to use the information to improve adherence and therapeutic alliance. An internist with HIV said, "If a patient asks me, I tell them if it seems to be serving a purpose, and I think it's worthwhile".

Doctors with HIV and other disorders also disclosed when frustrated at patients' complaints about matters that seemed, in comparison, to be minor. An internist with metastatic breast cancer said:

I became exasperated with this man, because all he did was complain: He had a horrible wife, was overweight, had diabetes, was depressed, his wife was having more children, and he was $\$ 300,000$ in debt. He felt that everything we suggested was not going to work ... I lost my patience and said, "Look, you want to know a real problem? I have a real problem". He was shaken, and I admit it was not very professional for me to do ... It did take him aback ... I said, "You have the ability to make yourself better, and I don't".

Similarly, an HIV-positive internist disclosed in order to discourage a patient who prematurely wanted to go on disability, "With $450 \mathrm{~T}$-cells, he was totally well ... I said, 'You've got more T-cells than I do! There's no reason you can't work"'.

Anxiety about these issues was high among doctors with HIV, who even worried in advance what to say if a patient asked. Some of these providers decided to tell patients only if the latter did in fact ask, and it felt "appropriate" to tell.

Yet alternatively, the stigma of HIV led some even to deny their illness to others. One doctor described an HIVinfected physician who refused to acknowledge an illness, and was surprised to be asked about it. "He denied all this: 'Why do people keep thinking I have AIDS?' He had been hospitalized for CMV!" 
At times, physicians with HIV, when asked about it by patients, might lie or mislead:

One patient said "I saw Dr. X and then he died; and then Dr. $\mathrm{Y}$, and a year later he died ... It may be me. I may be the kiss of death. Maybe I've got bad karma". Later, he said, "I just want to know: you're going to be around, right? You're not sick or anything?" I tacitly lied ... "No, I'm not sick".

Questions emerged of definitions of sins of omission and "partial" truths. Providers could misrepresent their conditions. An HIV-infected dentist worked in a small town, and many of his patients attended the same fundamentalist church as he and his family did. "My health was deteriorating, and people were asking. One patient asked me what was the matter." He would reply that he stopped work because of an auto accident-which he acknowledged was a misrepresentation, if not a lie.

"You haven't been looking very healthy lately. How come you really did quit practice?" I said, "Well, I had an automobile accident..." I do have back problems and allergy problems, and I would use both those excuses. All of those are somewhat true. The car accident was minor. But misrepresentation like that is akin to lying, although some people might not term it that way.

As a result of difficulties that may arise when patients do know a doctor's diagnosis, the information may be kept from a patient even after the doctor dies. Thus, after a doctor dies of AIDS, some patients found out last, often "only after the doctor drops dead".

Providers may feel that their diagnosis is not relevant to the clinical care they offer. Doctors with HIV also feared lawsuits from angry or fearful patients. The dentist said, "Probably the type of person that would sue would know that there would not be a factual scientific basis for it, but he or she could see dollar signs ..."

Concerns arose, too, that disclosure of HIV would hurt one's profession as a whole. ("I would be causing this sensationalism in the papers. I don't think it would be good for my profession: a bad advertisement").

None of these HIV-infected doctors engaged in exposure prone activities. Still, the question remained of how patients would react if they knew the diagnosis. A few doctors feared patients could then sue for psychological distress, even if the virus was not transmitted. Yet, some doctors pointed out, other illnesses could potentially harm patients more. A doctor's substance abuse, e.g., was potentially more dangerous than his or her HIV status. ("I'd much rather know if my doctor's had 3 drinks that morning before he takes me to the OR, than if he's got HIV. And surgeons who drink: that happens all the time").

\subsection{Collusion perpetuating silence}

Physicians and patients might collude to impede truthtelling, mutually not wanting to acknowledge a doctors' illness. Patients may want to think their doctor is healthy, since the very core of an ongoing doctor-patient relationship depends on stability — on a physician not becoming ill or dying. Another doctor with HIV said, "Some patients feel they can lean on you ... People want to assume the doctor's healthy. They can't quite imagine the doctor's not healthy".

Patients may even misinterpret an ill doctor's clues or hints about his or her health. When an internist with HIV informed patients that he was retiring to teach, many misconstrued the message:

I wrote a letter to introduce the subject that 3 months later, I'd be leaving, and that my associate was going to take over ... I thought: the next question they're all going to ask is "What are you going to do next?" So I just wrote that I'm going to retire, spend more time relaxing and traveling, and I'm going to work, spending some time teaching at the university. In my mind, that meant that I was going to be volunteering at the university to teach. Almost universally that phrase got interpreted as, "He is a professor at the university now. He doesn't have weekend call." They did not scratch their heads over this 45-year-old guy going from working 10-12 hours a day, to sitting in a cabana with a lemonade. They answered the question themselves by filling in the teaching at the university as a full-time job, with some free time to travel. I was amazed ... I'd had medical students in the office, so a lot of patients thought, "This is the perfect chance. He's just going to go up there and teach." They'd ask, "Can we still come and see you at the university?" I'd say, "Well, I won't be seeing patients". I found it easier not to challenge their concept of what was going on. Suddenly, it solved my problem. I dreaded the next 3 months of people saying: "What are you going to do?" Instead, everyone understood ... Most seemed to have dismissed entirely that I said I was retiring.

\subsection{Effects of disclosures on doctor-patient relationships}

At times with HIV and other disorders, a doctor's disclosure could benefit relationships with patients, engendering trust. To help a patient make a decision about treatment, e.g., a politically active physician with Hodgkin's disease told a homeless patient.

I was a human being with him. His life came out better as a result. He feels privileged to be privy to my secret, which says something about his value: that I trusted him to know. I didn't tell him it was a secret, but I entrusted him with something that I don't go around talking about.

Disclosure could improve the bonding between doctors and patients, making them feel like members of "the same club". The radiologist with skin cancer said,

In medicine... it's been a big plus rather than a negative to have cancer, because I deal with cancer patients a lot. I show them my big scar. 
Yet, patient knowledge of a doctor's illness could also skew the relationship. One doctor with HIV found it best not to have his health become too prominent in interactions.

The patients that do know usually ask me how I am and how I'm doing. They're very concerned. It's good, but seems to take the focus away from why they' re here. Making the focus of a patient's interaction on how I' $m$ doing just doesn't seem best.

\subsection{A model for examining complexities of doctor- patient interactions}

Fig. 1 presents a framework illustrating the complexities of these issues - the ways doctors and patients each make decisions, and the many possible combinations of types of communications that can ensue. Doctors and patients each must decide how to respond to the others' decisions about how to communicate. Clearly, numerous sets of mutual decisions are possible that can have different outcomes. Interactions concerning other types of issues (e.g., adherence or outcomes of risky treatment interventions) may similarly involve such inter-dynamics.

\section{Discussion and conclusion}

\subsection{Discussion}

These data shed light on the intricacies involved in balancing ethics with psychosocial aspects of doctor-patient relationships and communication. Patients faced dilemmas of whether to comment on a doctor's condition either implicitly or explicitly. Doctors faced critical questions of whether and what to disclose.

Overall, the broad types of issues that arose (i.e., whether, what, when, and to whom to disclose) were similar across diseases. Yet with HIV, given its stigma, infectivity, and epidemic nature, further questions emerged. With HIVinfected physicians, patients wondered if they had a right to know, and they sensed that to ask directly might be taboo. At times, patients appeared to feel ambivalent about knowing, since a doctor's illness threatened the core stability of an ongoing doctor-patient relationship. Doctors had to decide whether to tell patients without being asked, or to respond only if asked, and if asked, whether to tell the truth, lie or misrepresent the information. If divulged, the information could alter the doctor-patient relationship, strengthening or skewing it. Some patients only learned of a doctor's illness after the doctor has died. These complexities may appear, too, among physicians with other serious disorders besides HIV - particularly terminal or infectious diseases - and can be further investigated in future studies with larger samples.

These data raise larger important questions - e.g., about how ethical issues are approached and handled within the social and psychological nuances and complexities of doctor-patient relationships - often built on fragile trust. Doctors felt that a patient did not have a right to know their health status generally, unless it in some way threatened the patient's health. But dilemmas arose. What felt psychologically appropriate could conflict with what was considered ethical. Even if ethically unnecessary, disclosure may benefit the patient. Given competing trust and closeness developed over time, non-disclosure may also be awkward, since silence can create distance and tension. Hence, some doctors may disclose, even though not ethically required to do so. At times, doctors disclose, too, if it can help a patient coping. Disclosure may be more helpful and supported with regard to certain diagnoses or types of disorders than others. For example, former substance abusers often treat substance-abusing patients, and draw on personal experiences that may enhance effectiveness [28]. Physician disclosure can potentially strengthen patient-provider bonds, and thus trust and treatment alliances. Yet even here, clinicians face questions of how much, and what information to disclose. If a doctor discloses a positive HIV status, should he or she reveal the means of viral exposure (e.g., gay sexual activity)? Physician non-disclosure or misrepresentation may also skew the doctor-patient relationship, potentially undermining trust.

These decisions depend on the significance of the potential benefits and harms. Some psychological harm can occur without necessitating disclosure, but how much? Who should make these choices? Currently, physicians make these decisions alone, since initially they alone possess this information while their patients do not.

These data raise questions, too, of what information patients have a right to know, and how this issue should be decided. Patients may want or feel they have a right to information; and have such a right, if it is pertinent to their health [6]. But complications can emerge in these determinations, e.g., of whether such patient distress should ever trump the physician's right to privacy. Patients' claims involve their physical health, while physicians' claims may involve protection against potential stigma and discrimination. The degree of threat to a patient's health is key here. Patient death or serious physical harm clearly could trump a physician's right to privacy, yet patients may claim, without merit, that they suffer psychological distress from knowing that their physician is ill. Patients, even if remaining uninfected, have attempted to sue - though unsuccessfully, given the low degree of actual harm - HIV-infected physicians who had not disclosed that information [29].

Related dilemmas emerge if a doctor knows that he or she may or will not be able to provide long-term care to patients (e.g., due to retirement or change of jobs). Some patients might argue that they have the right to this information as well. But do they? When should physicians disclose such information: how much before leaving their job? These types of knowledge about a physician vary-from job change to serious illness to short life expectancy. At times, 
these types of information may benefit a patient. Especially in psychiatry, loss of a physician can distress psychologically fragile or vulnerable patients, and potentially exacerbate symptoms and decrease patient adherence, and hence health outcomes, if such patients also have medical problems. Patients' feelings of loss or betrayal (due to disease or its non-disclosure) could potentially affect attitudes, and future healthcare access and utilization. Further questions surface of whether physicians' symptoms (e.g., of fatigue or decreased concentration) could affect quality of care, necessitating disclosure as well. The effects on care may be small, but questions remain of who should make these disclosure determinations, and how.

This study has several potential limitations. The sample size may be small compared to certain studies, although for a qualitative study of this nature, it is large enough to provide insights into patterns of issues that emerge. Qualitative data of this sort can illuminate a wide range of important aspects of interpersonal issues that future quantitative research can explore in further detail with a larger sample. In this study, it was not feasible to interview physicians' patients directly; but these physicians' observations about patients' reactions appear to have a certain face validity. Future studies can investigate these areas further, including patient perspectives on these issues-which have not been explored in other research. These data focus on how physicians dealt with these issues at the time of interviews, at which point they had severe illness, rather than earlier, when their disease may have been milder. Many of these physicians had HIV, however, these data are thus in many ways unique and valuable for elucidating these issues in bold relief. Though most participants were white males, these issues did not appear to differ significantly here by gender or race. Nonetheless, future studies can investigate any possible such differences in greater detail.

\subsection{Conclusion}

Physician disclosures of their own HIV infection or other serious diagnoses to patients raise several critical issues, and suggest a framework - illuminating complexities of doctorpatient interactions - that can be used for examining other types of difficult or conflict-laden interactions as well, potentially enhancing understandings of conflicts between ethics and interpersonal aspects of doctor-patient relationships. These data illustrate the intricacies involved in applying ethical principles in the nuanced social and psychological contexts of doctor-patient relationships. Often, the ethical literature does not take fully into account the social contexts of suffering and emotional difficulties faced by doctors (e.g., when patients ask) and by patients (e.g., whether to ask).

\subsection{Practice implications}

These data have implications for guidelines for clinicians pondering how to approach these issues-to consider carefully pros and cons involved as described here, and to ensure that any disclosures benefit the patient. Physicians should not divulge all ailments to all patients, but should realize that patients may be anxious about these concerns, and view pros and cons of physicians' decisions differently than do these physicians themselves. Doctors may decide not to disclose certain information, but should then be aware of the impact of these decisions on patients. An ethically justified decision not to disclose may still impact patients and relationships. Similarly, despite ethical justification of reticence, doctors may volunteer information about their health, but not be as aware as they should of the potential risks and benefits of doing so. Critical, finegrained questions also remain of not only whether, but what, when, and to whom to disclose. Future research needs to probe further the effects of each of these decisions. A physician who decides that disclosure is not appropriate, in answer to a patient's query, could respond not by ignoring or evading the question, but by acknowledging the query and alluding to the decision involved. For example, the physician could respond, as suggested in these data: "We could discuss my health, but I think it makes more sense to talk about your health issues here today, rather than my own." Physicians and patients each hold implicit definitions of "appropriate" communication, and these may or may not coincide. Clarification and increased awareness and attention regarding the intricacies and impact of these issues can help both doctors and patients in knowing how best to address their respective fears and concerns, in order to most strengthen doctor-patient relationships.

\section{Acknowledgements}

The authors would like to thank Renée C. Fox, Mary DuVernay, Frank Griggs, Shaira Daya, Daniel Fishman, Brian Kristoff, and Jennifer Hersh, for assistance with this project. This project was funded by the Arthur Vining Davis Foundations, the Picker-Commonwealth Scholars Program, the Commonwealth Fund, and in part through the NIMH (Grant K08-MH-1420-01).

\section{References}

[1] Remien R. Adhering to HIV combination therapy: the role of the pharmacist. Pharmacy Times 1998;28-37.

[2] Freeman J, Loewe R. Barriers to communication about diabetes mellitus: patients' and physicians' different view of the disease. J Family Pract 2000;49:507-12.

[3] Quill T. Recognizing and adjusting to barriers in doctor-patient communication. Ann Intern Med 1989;111:51-7 [review].

[4] Klitzman R. Being positive: the lives of men and women with HIV Chicago, IL: Ivan R. Dee, Inc.; 1997.

[5] Klitzman R, Bayer R. Mortal secrets: truth and lies in the age of AIDS Baltimore, MD: Johns Hopkins University Press; 2003.

[6] Gostin L. HIV infected physicians and the practice of seriously invasive procedures. Hastings Center Rep 1989;19:32-9. 
[7] Parsons T. Illness and the role of the physician: a sociological perspective. Am J Orthopsychiatry 1951;21:452-60.

[8] Goffman E. Stigma: notes on the management of spoiled identity Englewood, NJ: Prentice-Hall; 1963.

[9] Hahn R. Between two worlds: physicians as patients. Med Anthrop Quart 1985;87-98.

[10] Stoudemire A, Rhoads J. When the doctors needs a doctor: special considerations for the physician-patient. Ann Intern Med 1983;98:654-9.

[11] Pinner M, Miller B, editors. When doctors are patients. New York: W.W. Norton; 1952.

[12] Mandell H, Spiro H, editors. When doctors get sick. New York: Plenum Press; 1987.

[13] Sacks O. A leg to stand on New York: Harper Collins; 1989.

[14] Mullan F. Seasons of survival: reflections of a physician with cancer. N Engl J Med 1985;313:270-3.

[15] Heymann J. Equal partners Boston: Little Brown; 1995.

[16] Aoun H. When a house officer gets AIDS. N Engl J Med 1989;321:693-6.

[17] Aoun H. From the eye of the storm, with the eyes of a physician. Ann Intern Med 1992;116:335-8.

[18] Beach MC, Roter D, Larson S, Levinson W, Ford DE, Frankel R. What do physicians tell patients about themselves? J Gen Intern Med 2004;19:911-6.
[19] Beach MC, Roter D, Rubin H, Frankel R, Levinson W, Ford DE. Is physician self-disclosure related to patient evaluation of office visits? J Gen Intern Med 2004;19:905-10.

[20] Frank E, Breyan J, Elon L. Physician disclosure of healthy personal behaviors improves credibility and ability to motivate. Arch Fam Med 2000;9:287-91.

[21] Tinsley JA, Mellman LA. Patient reactions to a psychiatrist's pregnancy. Am J Psychiatry 2003;160:27-31.

[22] Peterson Z. More than a mirror: the ethics of therapist self-disclosure. Psychotherapy: Theory/Res/Pract/Training 2002;39:21-31.

[23] Tillman JG. Psychodynamic psychotherapy, religious beliefs, and selfdisclosure. Am J Psychotherapy 1998;52:273-86.

[24] Dilts S, Clark C, Harmon R. Self-disclosure and the treatment of substance abuse. J Substance Abuse 1997;14:67-70.

[25] Gabbard GO, Nadelson C. Professional boundaries in the physicianpatient relationship. J Am Med 1995;273:1445-9.

[26] Fay A. Letter to the editor. J Am Med 1995;274:1345-6.

[27] Strauss A, Corbin J. Basics of qualitative research-techniques and procedures for developing grounded theory Newbury Park: Sage Publications; 1990.

[28] Dilts S, Clark C, Harmon R. Self-disclosure and treatment of substance abuse. J Substance Abuse Treatment 1997;14:67-70.

[29] Court retracts mandate that physicians must disclose HIV. AIDS Policy Law 1999;14:5. 